\title{
El Aprendizaje-Servicio como estrategia metodológica en la Universidad
}

\section{Service-learning as a methodological strategy at University}

\author{
Margarita R. RODRÍGUEZ GALLEGO \\ Universidad de Sevilla
}

Recibido: Enero 2013

Aceptado: Abril 2013

\section{Resumen}

El presente trabajo describe la investigación llevada a cabo con estudiantes del Grado de Pedagogía, de la Facultad de Ciencias de la Educación de la Universidad de Sevilla (España), en la asignatura Didáctica General, con el objetivo de vincular teoría- práctica a través de una metodología de Aprendizaje-Servicio (Service-Learning). Este trabajo ha sido aprobado por el Vicerrectorado de Ordenación Académica, como reconocimiento por la participación en actividades culturales, deportivas, de representación estudiantil o solidarias y de cooperación, de la Universidad de Sevilla ${ }^{1}$. Los resultados de la evaluación realizada, por el Centro educativo, estudiantes universitarios y Universidad son muy alentadores. En la autoevaluación realizada para detectar las posibilidades de mejora e institucionalización del AprendizajeServicio hemos constatado que debemos implantar y fortalecer docencia estructurada de primer, segundo y tercer ciclo (itinerarios de grados, programas de doctorado, títulos propios,...) destinada a la formación de los estudiantes, dotando a las existentes de contenidos teóricos y prácticos aplicables al Aprendizaje-Servicio. Estos hallazgos enfatizan la importancia de esta metodología para la mejora de los aprendizajes académicos en la Universidad y el fortalecimiento de un compromiso ciudadano con la Comunidad.

Palabas clave: Aprendizaje-Servicio, metodología, universidad, prosocialidad.

\begin{abstract}
This paper describes the experience performed with students on an education programme, in the Faculty of Educational Sciences at the University of Seville (Spain). The subject is called General Teaching and its objective is to improve the relation between practices and theory through a Service-Learning methodology. The project has been approved by the University of Seville and has been recognized for its involvement in cultural, sport, student representation, solidarity and cooperation activities in the university environment. The results of the

${ }^{1}$ Agradecimiento al Vicerrectorado de Ordenación Académica de la Universidad de Sevilla.


assessments made by the school, college and university are very encouraging. In the selfevaluation carried out to identify areas that develop Service-Learning methodology we have found that it is necessary to implement and strengthen teaching structure at all university levels (grades itineraries, undergraduate programs, doctoral programs, ...) addressed to student training. These findings emphasize the importance of methodology to improve academic learning at University and strengthen citizen engagement with the Community.

Keywords: Service-Learning, methodology, university, prosociality.

El objetivo de este trabajo es dar a conocer la investigación desarrollada a través de la metodología de Aprendizaje-Servicio (ApS), en los estudios de Grado de Pedagogía de la Facultad de Ciencias de la Educación de la Universidad de Sevilla.

En este sentido, para justificar el estudio exponemos conceptos fundamentales relacionados con el Aprendizaje-Servicio, algunas experiencias e investigaciones desarrolladas en el ámbito nacional e internacional, así como el trabajo que vienen desarrollando departamentos universitarios, grupos y organismos institucionales que promueven este tipo de enseñanza en distintos países. Estamos convencidos que el ApS es una buena estrategia metodológica al combinar la formación teórica de una asignatura con la participación de los estudiantes en proyectos en los que prestan un servicio gratuito a la sociedad. Las experiencias de aplicación de esta metodología ponen de manifiesto una comprensión más amplia y profunda del contenido del curso en el que se realiza, desarrolla el sentido de compromiso ciudadano y mejora el conocimiento de sí mismo y de su posición en la comunidad. Además de adquirir competencias y habilidades personales que son difíciles de alcanzar por otros métodos. Las aplicaciones prácticas realizadas en el ámbito universitario avalan que el ApS es una estrategia didáctica poderosa y que se puede estructurar de múltiples maneras, en proyectos a nivel de Universidad, Facultad/Facultades y asignaturas concretas.

Finalmente, presentamos la investigación desarrollada por los estudiantes universitarios, en un Centro de Educación Infantil y Primaria de Sevilla, con resultados muy gratificantes para todos los sectores implicados.

\section{Aprendizaje-Servicio (ApS): delimitación conceptual}

El Aprendizaje-Servicio ha tenido gran impulso en los últimos años, primero en los Estados Unidos y Argentina, después en Reino Unido y el resto de Europa, como medio para promover la participación de los jóvenes y adolescentes al servicio de la comunidad tanto en la enseñanza media como superior (Annette, 2000). La expresión inglesa Service-Learning viene a subrayar el enfoque educativo que estas actividades de servicio tienen. En ellas los estudiantes aprenden y maduran mediante la participación activa en experiencias de servicio organizadas para adquirir conocimientos, siendo integradas en el currículum académico, a la vez que cubren necesidades sociales. Se trata de una forma de educación experiencial en la que los estudiantes se comprometen en actividades de ayuda a la comunidad al tiempo que facilita el aprendizaje de una asignatura y el desarrollo de competencias profesionales. 
El ApS es "una actividad o programa de servicio solidario protagonizado por los estudiantes, orientado a atender eficazmente las necesidades de una comunidad, y planificada de forma integrada con los contenidos curriculares con el objetivo de optimizar los aprendizajes" (Tapia, 2008, p. 43).

Es importante diferenciar esta propuesta de otro tipo de iniciativas con clara intencionalidad solidaria pero dudoso impacto educativo, así como de experiencias claramente educativas pero con escaso impacto solidario. El aprendizaje-servicio no es cualquier tipo de voluntariado, ni un practicum sin más, pues incluye tanto objetivos de servicio como objetivos de aprendizaje. En la Tabla 1 ilustra las diferencias entre ApS, voluntariado y servicio comunitario.

\begin{tabular}{|l|l|}
\multicolumn{1}{|c|}{ APRENDIZAJE SERVICIO } & \multicolumn{1}{c|}{$\begin{array}{c}\text { VOLUNTARIADO Y SERVICIO } \\
\text { COMUNITARIO }\end{array}$} \\
\hline $\begin{array}{l}\text { Enfoque pedagógico-solidario y metodología de } \\
\text { enseñanza-aprendizaje }\end{array}$ & Enfoque pedagógico-solidario \\
\hline Objetivos de servicio y objetivos de aprendizaje & Objetivos de servicio \\
\hline Formación profesional y formación ciudadana & Formación ciudadana \\
\hline Requiere fases de preparación, acción y reflexión & Comprende la actividad misma \\
\hline $\begin{array}{l}\text { Existen normas establecidas para el desempeño del } \\
\text { servicio }\end{array}$ & No existen normas establecidas \\
\hline $\begin{array}{l}\text { Requiere planteamiento anticipado a la fecha del } \\
\text { servicio }\end{array}$ & Puede ocurrir en cualquier momento \\
\hline $\begin{array}{l}\text { Debe realizarse con la supervisión adecuada de un } \\
\text { adulto }\end{array}$ & Puede realizarse sin supervisión de un adulto \\
\hline Certificado de acreditación del servicio & No es un requisito \\
\hline
\end{tabular}

Tabla 1. Diferencias entre ApS y voluntariado y servicio comunitario.

El ApS, como hemos planteado anteriormente, es un método de enseñanza que, concretamente en el ámbito de Educación Superior, busca vincular el aprendizaje del estudiante con el servicio a la comunidad generando beneficios en tres ámbitos:

- Currículum académico, promueve una mayor formación práctica y la reelaboración de los contenidos teóricos para hacerlos más pertinentes al contexto social y económico para el desempeño profesional,

- Formación en valores, en aspectos como prosocialidad, la responsabilidad social, la solidaridad, la pertinencia de la actividad profesional, entre otros; ayudando a la formación para la ciudadanía de los profesionales que en el futuro tendrán un destacado papel en la sociedad,

- Vinculación con la comunidad, puesto que la intervención surge de la demanda explícita de la sociedad y promueve la intervención de carácter profesional sobre una problemática social real. 
El modelo ApS constituye un ejercicio de unión de aspectos fundamentales que tradicionalmente están por separado en las instituciones educativas: la teoría con la práctica, el aula con la realidad, la formación con el compromiso y la cognición con la emoción (Butin, 2006; Manzano, 2010).

Como plantea Manzano (2010) para que podamos hablar de ApS es necesario que coexistan cuatro características sobresalientes: aprendizaje académico, orientación hacia la transformación social, diálogo horizontal con la comunidad y peso presencial de los estudiantes.

Martínez (2007) argumenta que una formación universitaria de calidad no puede separar la formación profesional de la formación ciudadana y ofrece tres razones: la primera relacionada con el concepto actual de formación universitaria; la segunda con el objetivo de cohesión social y no sólo de competitividad que debería tener como misión la universidad hoy; y la tercera derivada de las investigaciones sobre desarrollo moral y aprendizaje ético.

Por tanto, una práctica de ApS de calidad debe implicar un aprendizaje riguroso, vinculado simultáneamente a una acción solidaria planificada, procurando impactar de forma positiva en la vida de una comunidad.

Por todo ello, el ApS resulta ser una metodología y una verdadera filosofía de la educación y el modelo formativo de las Universidades debe cambiar para promover, en su práctica y en sus espacios de convivencia y trabajo, situaciones de implicación con la comunidad. El ApS es un descubrimiento y puesta en valor de buenas prácticas que las buenas instituciones y las buenas entidades sociales desarrollan desde hace tiempo (Batlle, 2011).

\section{Propuestas desarrolladas en el ámbito nacional e internacional}

Aunque la literatura sobre ApS es muy abundante en ejemplos de aplicaciones prácticas, nos centraremos exclusivamente en experiencias desarrolladas en Educación Superior.

A día de hoy, multitud de Universidades, colleges, departamentos y profesores se han acogido al ApS y, paralelamente, se ha ido evolucionando desde el concepto tradicional de extensión universitaria al de responsabilidad social universitaria. Queremos aclarar que no todas las iniciativas de responsabilidad social universitaria tienen por qué ser de Aprendizaje-Servicio, hay programas institucionales desarrollados por equipos docentes o profesionales sin participación de estudiantes, pero todos los programas de Aprendizaje-Servicio son, por definición, una manifestación de responsabilidad social (Tapia, 2008).

En el contexto anglosajón, el concepto de ApS ha sido desarrollado tanto desde la investigación como desde la práctica educativa y comunitaria. Prueba de ello es la existencia de departamentos de aprendizaje-servicio en la mayoría de universidades estadounidenses (Maryland, Colorado, Indiana, San Francisco o Duke, entre otras). 
Por otro lado, también cabe destacar en este sentido las aportaciones que han realizado diversos grupos de desarrollo teórico y práctico del concepto como el National Service Learning Partnership, con una sección dedicada a la investigación en este campo, o la International Association for Research on Service Learning and Community Engagement, que organiza las conferencias internacionales en este ámbito. También existen publicaciones especializadas en el área como, Partneships: A Journal of Service-Learning \& Civil Engagement o The International Journal of ServiceLearning in Engineering.

Hay organismos institucionales que promueven este tipo de enseñanza, vinculada al voluntariado y la acción cívica (como el caso de Learn and Serve America http://www.learnandserve.gov/), así como redes de universidades o de organizaciones de la sociedad civil (como el caso de Campus Compact http://www.compact.org/) que promocionan el voluntariado estudiantil y el ApS. Se trata de una coalición nacional formada por unos 1100 Colleges y universidades (públicas y privadas) procedentes de más de 50 Estados, además de algunos miembros internacionales que, con sus rectores a la cabeza, se han comprometido en el logro de la misión cívica en la Educación Superior. Para hacer efectivos esos fines, Campus Compact promueve el servicio a la comunidad como ayuda a los estudiantes para adquirir disposiciones y habilidades cívicas, fomenta la relación entre los campus y sus entornos, y ofrece el apoyo necesario a los profesores que buscan integrar temas de interés público en su docencia e investigación. En el mismo contexto se encuentra la Asociación Internacional de Investigaciones sobre ApS: International Association for Research on Service-Learning and Community Engagement (http://www.researchlce.org/)

Por último existen, también centros de investigación y de promoción del ApS en el marco de diferentes universidades. Un ejemplo interesantes es el Service-Learning Research and Development Center de la Universidad de California (http://gse.berkeley.edu/research/slc/).

En el ámbito iberoamericano, también hay un gran desarrollo teórico y metodológico de este enfoque, con algunas experiencias de interés en la investigación y el desarrollo del ApS. Argentina es junto con EE.UU., uno de los principales motores en este tema con el Centro Latinoamericano de Aprendizaje Servicio Solidario (CLAYSS, http://www.clayss.org/). Este centro estableció las bases de la creación en 2005 de la Red Iberoamericana de Aprendizaje y Servicio Solidario, integrada por asociaciones y entidades de Latinoamérica, Estados Unidos y España.

En Europa, Lernen durch Engagement, es la organización alemana que promueve el ApS. Movisie es una veterana organización holandesa que en este momento está contribuyendo a reconvertir prácticas de voluntariado en Aprendizaje-Servicio. Aprendre en s'engageant es la organización suiza para el impulso del ApS. Community Service Volunteers es una veterana organización británica centrada sobre todo en la promoción del voluntariado.

En nuestro país se ha creado una Red Española de Aprendizaje-Servicio (http://www.aprendizajeservicio.net/). Se trata de una Red de redes territoriales y 
sectoriales inspirada en los valores del ApS: confianza, generosidad, cooperación y respeto (Batlle, 2012).

El ámbito de la investigación en este tema es muy amplio y abarca desde el ApS vinculado con la educación moral (Annette, 2005), aspectos que favorecen o dificultan la introducción de proyectos ApS (Galambos \& Kozma, 2005), relación entre el ApS y la adquisición de competencias (Murphy, 2010), efectos que el servicio tiene en los estudiantes universitarios (Holdsworth \& Quinn, 2010), como estrategia metodológica (Bednarz et al., 2008; Reinders, 2010), impacto que el ApS produce tanto en el aprendizaje como en el desarrollo de competencias y actitudes en los estudiantes universitarios (Tapia, 2000; Furco, 2005) hasta el desarrollo de programas y actividades de ApS (Hollander et. al., 2001; Buchana et.al., 2002; Furco \& Billing, 2002).

En las Universidades españolas, como el caso de la Universidad de Navarra se ha profundizado en el análisis bibliográfico sobre el concepto "Service-Learning" (Martínez Odría, 2005). En la Universidad de Barcelona se han desarrollado 4 experiencias: Derecho al Derecho, Educación física y educación social, Amigos y amigas de lectura y Prácticas de cooperación. Las tres primeras se han implantado en la Universitat de Barcelona y la última en la Universitat de Vic (Cataluña) con muy buenos resultados y expectativas (Martínez, 2007). En la Universidad de Sevilla se están desarrollando proyectos de ApS con propuestas de investigación-acción participativas para resolver problemas de vivienda en la asignatura Análisis de Datos de la Facultad de Psicología (Manzano, 2010).

Es importante resaltar que cuando las prácticas de ApS son promovidas como parte del proyecto pedagógico-solidario de una Universidad el grado de institucionalización es muy superior y es posible incluirlas en los planes de estudio para adecuarlos a este tipo de prácticas, revisar los marcos normativos y de difusión de las prácticas con mayor alcance que en otros casos. Éste ha sido el motor que ha impulsado nuestra investigación.

\section{Metodología}

En este trabajo de investigación presentamos un modelo implantado en el Grado de Pedagogía de la Facultad de Ciencias de la Educación, con la aprobación del Vicerrectorado de Ordenación Académica de la Universidad de Sevilla (España), en la asignatura Didáctica General. La implantación de este modelo, en esta asignatura, comenzó en el curso académico 2009-2010, con muy buenos resultados académicos y de servicio, pero la sistematización se materializa en el curso académico 2011-2012.

\section{Finalidad y objetivos}

Teniendo en cuenta los antecedentes y el estado de la cuestión expuesta, con esta investigación pretendemos vincular teoría- práctica a través de una metodología de Aprendizaje-Servicio (Service-Learning). De modo concreto, los objetivos son los siguientes: 
- Diseñar un plan de acción que permita la incorporación de la metodología ApS en la asignatura Didáctica General.

- Determinar, una vez implementada en la práctica, si el ApS es una buena estrategia metodológica según la opinión de los sectores implicados.

\section{Muestra}

La selección de la muestra ha sido casual o incidental pues se ha trabajado con todos los grupos de primer curso del Grado de Pedagogía, profesoras de la Facultad, profesionales de un Centro de Educación Infantil y Primaria (CEIP) de Sevilla y un responsable del Vicerrectorado de Ordenación Académica. La distribución ha quedado de la siguiente forma: 360 estudiantes de primer curso del Grado de Pedagogía, matriculado en el curso académico 2011-2012, 8 estudiantes-servicio, 2 profesoras que imparten la asignatura Didáctica General, 20 profesionales del Centro de Infantil y Primaria y una persona en representación de la Universidad. La distribución de la muestra queda recogida en la Tabla 2.

\begin{tabular}{|l|c|}
\hline \multicolumn{1}{|c|}{ Perfil } & (n) \\
\hline Estudiantes Facultad & 360 \\
\hline Estudiantes-servicio & 8 \\
\hline Profesoras Facultad & 2 \\
\hline Profesores CEIP & 20 \\
\hline Representante Universidad & 1 \\
\hline
\end{tabular}

Tabla 2. Distribución de los participantes en la investigación.

\section{Diseño de la investigación}

Nuestro diseño de investigación es etnográfico pues nuestro fin es descubrir lo que sucede cotidianamente, tanto en las aulas de la Facultad como en las aulas del CEIP, aportando datos significativos, de la forma lo más descriptiva posible, para interpretarlos, comprenderlos y poder intervenir adecuadamente en nuestra realidad particular. El diseño es emergente y en cascada, lo hemos ido elaborando a medida que ha avanzado la investigación.

La investigación de campo ha sido realizada en equipo por 2 profesoras de la Facultad, 20 profesionales del CEIP y 8 estudiantes-servicio que han participado en este estudio. 


\section{Fases}

El proceso ha pasado por las siguientes fases de construcción:

Diagnóstico: La investigación comenzó con la realización de un diagnóstico institucional para evaluar la necesidad de contar con recursos humanos disponibles; y un diagnóstico local para dar a conocer las problemáticas y necesidades reales de la Comunidad en la que se lleva a cabo el proyecto, así como agencias e instituciones públicas y privadas que pudiesen colaborar en el mismo.

Hay que decir que, el 95\% del alumnado del CEIP es de etnia gitana y vive en una situación de desventaja social. La mayoría de los estudiantes del centro cuenta con un desfase curricular entre uno y dos cursos escolares. Las características más sobresalientes de la población son las que se recogen en la Tabla 3.

\begin{tabular}{|l|}
\hline Población marginada y excluida socialmente \\
Mayoría étnica gitana \\
Población procedente de suburbios, desahucio, existiendo problemas de desarraigo, \\
integración,... etc. \\
Relaciones interpersonales conflictivas: \\
Ambito familiar: malos tratos, agresividad jerarquizada, hacinamiento.... \\
Ambito vecinal: agresividad hacia el entorno, vandalismo urbano..... \\
Inseguridad ciudadana, delincuencia... \\
Consumo y tráfico de drogas así como otras actividades ilegales \\
Insuficiente conciencia higiénico-sanitaria, que conlleva graves problemas de salud y \\
Analfabetismo y resistencia a acceder a niveles básicos de enseñanza, bajo nivel de instrucción, \\
falta de información y bajo nivel cultural \\
Alto índice de paro en la población joven y adulta, profesiones sin cualificar, economía sumergida, \\
ocupaciones marginales, que conlleva bajo poder adquisitivo y situaciones de pobreza, llegando hasta la \\
falta de medios necesarios para la supervivencia (alimentos, ropa, calzado...) \\
\hline Deficiente escolarización con: alto índice de absentismo, fracaso escolar y abandono escolar \\
\hline Tabla 3. Carativas para la calidad de vida
\end{tabular}

Tabla 3. Características de la población del Centro de Educación Infantil y Primaria.

Para reclutar a estudiantes del grado de Pedagogía, planeamos una reunión en el mes de octubre, el equipo directivo del CEIP y las profesoras de la Facultad de Ciencias de la Educación, con el objetivo de dar a conocer el proyecto.

Coordinación inicial: Elaboramos un borrador del proyecto, por parte del profesorado del CEIP y las profesoras de la Facultad, donde quedó recogida la colaboración de los estudiantes y entidades. El principal recurso humano con el que 
cuenta el CEIP ha sido el claustro de profesores así como la colaboración asegurada de diferentes entidades: Asociación Entre Amigos, Asociación Unión Romaní, Facultad de Psicología, Facultad de Ciencias de la Educación, Residencia Universitaria Flora Tristán (varios becarios han colaborado en el centro en diferentes actividades), Asociación de Mujeres Gitanas Akerdhi Tromipén, Equipo de Trabajo Social y familias.

Los profesores del CEIP consideraron deseable y aconsejable que los estudiantes implicados en el servicio recibieran una formación inicial para la adquisición de los conocimientos necesarios en el desempeño de su labor en el centro. Para ello tuvieron que asistir al inicio del curso a las Jornadas de Sensibilización, organizadas por el Centro de Profesorado (CEP) de Sevilla, para conocer qué es y cómo funciona una Comunidad de Aprendizaje (Barrio, 2005). A su vez el centro ha distribuido entre los estudiantes universitarios un documento con sus líneas generales de actuación a nivel pedagógico y organizativo.

Diseño: Cada estudiante ha elegido el horario que mejor se ha ajustado a su disponibilidad, de este modo la franja horaria en la que han colaborado los estudiantes de la Facultad ha sido de lunes a viernes de 9:00 a 14:00 horas y de lunes a jueves de 15:00 a 16:00 horas. Cada estudiante ha cubierto 75 horas (computable con 3 créditos ECTS), desde diciembre hasta mayo y las actividades realizadas han sido trabajo en grupos interactivos, proyectos, biblioteca tutorizada y tertulias literarias dialógicas.

El temario de la asignatura Didáctica General ha quedado reforzado con el aprendizaje de estrategias metodológicas teóricas y su aplicación práctica con el servicio ofrecido en el CEIP. De este modo, los cuatro grupos de la asignatura se han visto favorecidos por la experiencia vivida por 8 compañeros voluntarios.

Ejecución: Los estudiantes-servicio han aprendido como planificar e implementar los grupos interactivos en las aulas del CEIP. El grupo clase se ha dividido en grupos pequeños de trabajo, cada grupo ha estado tutorizado por una persona adulta (otro docente, ApS, voluntariado, familiares, exalumnos...) facilitando la creación de nuevos espacios de trabajo orientados a la aceleración del aprendizaje comunicativo y cooperativo mediante un seguimiento individualizado y grupal. Los grupos interactivos se han concretado, al menos, en dos sesiones semanales de una hora y cuarenta y cinco minutos en todos los niveles educativos, en función del número de estudiantes universitarios que han acudido al centro. En un primer momento se llevaron a cabo grupos interactivos en las áreas de Matemáticas y Lengua, y en menor medida se ha extendido a otras áreas como Lengua Extranjera (Inglés).

Para la puesta en práctica de esta experiencia hemos contado con todo el profesorado del centro (tutores/as y especialistas) con la intención de optimizar los recursos disponibles y garantizar la presencia en cada aula de dos profesores del centro y un estudiante. Esta metodología ha tratado de no separar al alumnado en función de sus dificultades, sino de potenciar sus aprendizajes dentro del aula. Los grupos interactivos han sido heterogéneos, tanto en cuestión de género como en nivel de aprendizaje $\mathrm{u}$ origen cultural. Todos los grupos rotaron por las diferentes actividades que se han organizado para cada sesión y cada uno ha estado a cargo de un 
universitario. En cada grupo, el estudiante-servicio ha seguido con mayor atención el desarrollo del trabajo de cada uno de los alumnos, identificando las dificultades y fomentando que lleguen a resolverlas ayudándose entre sí y enriqueciendo las interacciones. El docente tutor del aula ha sido quien ha programado las actividades didácticas con relación a los objetivos curriculares que era necesario conseguir, ha dinamizado la sesión y ha coordinado los tiempos. De esta manera en una misma dinámica se dieron dos desarrollos: el de los valores como la solidaridad o la tolerancia hacia la diversidad y el de aprendizaje instrumental.

Otra propuesta, en la que han colaborado los estudiantes-servicio, ha sido la metodología de trabajo por Proyectos para el área de Conocimiento del Medio, fundamentalmente, pero dado el carácter globalizador de los mismos, se ha trabajado también objetivos y contenidos de las demás áreas que configuran el currículum de Educación Infantil y Primaria. El desarrollo de un Proyecto de trabajo (investigación) es un proceso organizado y flexible que se ha articulado en base a la realización de una serie de acciones y actividades englobadas en las fases de planificación, búsqueda, estructuración y evaluación.

La biblioteca tutorizada ha permitido la ampliación del tiempo de aprendizaje en el centro. La biblioteca abre sus puertas fuera del horario lectivo por parte de profesores colaboradores, familias, estudiantes ApS...para que acudan a ella aquéllos alumnos con dificultades de aprendizaje ligadas a la lectura y escritura con el objeto de una atención especializada que les permita la superación de sus dificultades. Además de estos alumnos, durante el horario de biblioteca tutorizada, han acudido a ella y han hecho uso alumnos, exalumnos, sus familiares, ya sea para leer un libro, para hacer los deberes, para consultar dudas... La viabilidad de la biblioteca tutorizada ha venido por el compromiso de un grupo amplio de profesores, estudiantes ApS y voluntarios que han permanecido en el centro de 15:00 a 16:00 horas para que la biblioteca pudiera abrirse. El horario se ha ampliado con la ayuda de los estudiantes-servicio de la Facultad.

Las tertulias literarias dialógicas han ido dirigidas a toda la comunidad para acercar a las personas sin formación académica a la literatura clásica universal. En Infantil y primer ciclo de Primaria no han sido en sí tertulias dialógicas, sino que se ha comentado los diferentes libros que se han ido leyendo en clase. En el segundo y tercer ciclo los alumnos han elegido y leído un libro de reconocido prestigio en la Literatura Infantil. Todos los cursos han tenido tres momentos de $1 / 2$ hora de lectura en horario lectivo. En la hora de Lengua o tutoría se ha dedicado un tiempo específico para la tertulia, en un primer momento los alumnos se han mostrado reacios a participar, pero en poco tiempo han participado activamente y se han contagiado el entusiasmo por leer y expresar. En las sesiones de tertulia se ha dialogado sobre lo leído y el párrafo que más ha llamado la atención de cada lector.

Todas las intervenciones anteriores se han visto reforzadas con las sesiones de clase teóricas en la Facultad. Las profesoras han guiado el proceso a través del temario de la asignatura Didáctica General y los estudiantes implicados en ApS han ido describiendo y exponiendo, al resto de los compañeros del grupo clase su experiencia, expectativas y motivaciones aprendidas a través del contacto con la realidad. Los estudiantes 
implicados han redactado un informe describiendo su experiencia e incluyendo los aprendizajes derivados del estudio y de la reflexión en el aula. Dicho informe ha sido colgado en la plataforma institucional de la asignatura.

La participación de los estudiantes en el marco de la investigación ha consistido, en prestar un servicio a la comunidad educativa, tomar consciencia y exponer los aprendizajes conseguidos, conjugando los conocimientos disciplinares trabajados en la clase de Didáctica General con el servicio realizado en las aulas del CEIP.

Reflexión: La reflexión ha sido un proceso esencial que ha estado incluida en todas las fases del proyecto. Los estudiantes-servicio, profesionales del CEIP y profesoras de la Facultad han llevado un registro (cuaderno de campo) de la experiencia en cada intervención educativa. Junto con esta reflexión individual se han llevado a cabo entrevistas semiestructuradas para compartir opiniones con todos los sectores implicados en la investigación.

Evaluación: Para evaluar los resultados se ha tenido en cuenta la observación participante, entrevistas semiestructuradas a 20 profesionales del CEIP, 2 profesoras de la Facultad, 8 estudiantes ApS, 20 estudiantes de la asignatura Didáctica General seleccionados al azar y 1 representante de la Universidad.

De este modo, se han realizado entrevistas cruzadas de estudiantes, profesores y Universidad. En total se han realizado 51 entrevistas. Además de una autoevaluación por sector para ver las posibilidades de institucionalización definitiva y mejora del ApS.

Cierre y celebración: Una vez finalizada la experiencia se entregaron diplomas acreditativos a los alumnos que han realizado el servicio y se reconoció la labor de los profesores del centro y Facultad, así como de las entidades participantes.

\section{Instrumentos}

En la observación participante no utilizamos categorías de registro de datos ni unidades de análisis preconcebidas, sino las informaciones surgidas en los procesos de acción en cada una de las fases del diseño de la investigación. De esta manera, los etnógrafos, en este caso profesionales del CEIP, profesoras de la Facultad y estudiantes- servicio, hemos participado abiertamente, de la vida cotidiana de personas durante un cuatrimestre, viendo lo que pasa, escuchando lo que se dice, preguntando; o recogiendo todo tipo de datos accesibles para poder arrojar luz sobre la experiencia vivida. Nuestro método de registro han sido las notas de campo y las grabaciones de vídeo y audio.

Para las 51 entrevistas semiestructuradas hemos utilizado la técnica de análisis de contenido con la identificación, selección y estructuración de las categorías y subcategorías para cada uno de los grupos implicados en la investigación. El guión de la entrevista ha quedado estructurado de la siguiente forma:

- ¿El aprendizaje-servicio vincula explícitamente un servicio a la comunidad con un aprendizaje significativo?. 
- ¿El ApS proporciona estrategias para que los individuos reflexionen acerca de lo que hicieron durante la actividad de servicio y la conexión con el aprendizaje en la Facultad?.

- ¿El ApS promueve la formación en valores?. ¿De qué tipo?.

- ¿El Aps promueve el crecimiento personal?.

- ¿El ApS refuerza los lazos sociales con la comunidad?

En la autoevaluación se ha pedido a todos los colectivos implicados establecer mejoras pedagógicas, mejoras relacionales y mejoras organizativas del ApS.

\section{Resultados}

El sistema de codificación de las entrevistas se ha configurado a partir de las transcripciones de los textos grabados, identificando núcleos de ideas con sentido completo (Gürtler y Huber, 2007). El análisis de contenido se ha realizado por medio del programa Atlas.Ti 6.2. Nuestro enfoque para el análisis de datos cualitativos ha sido interpretativo y no lexicométrico. Para ello realizamos la tarea básica de reducción de la información a través de categorizaciones y codificaciones. El sistema de categorías ha sido inductivo pues al examinar los datos de la entrevista nos preguntamos por el tópico más adecuado capaz de cubrir cada unidad, generando así la categoría y subcategorías.

Para la validación del sistema de categorías han participado 10 jueces, profesores de la Facultad de Ciencias de la Educación del departamento de Didáctica y Organización Educativa, que han valorado el grado de concordancia en función del índice kappa para cada categoría planteada.

El coeficiente kappa refleja la concordancia inter-observadores en ,81. Podemos interpretar este coeficiente como muy bueno $(0,8-1)$.

Estimated Kappa, Asymptotic Standard Error, and Test of Null Hypothesis of 0 Population Value

\begin{tabular}{|c|c|c|c|}
\hline Kappa & ASE & Z-Value & P-Value \\
\hline 08132199 & ,02074834 & 30,42759132 & ,00000000 \\
\hline
\end{tabular}

Tabla 4. Índice estimación kappa categorías y subcategorías.

Presentamos los resultados obtenidos agrupados por categorías (currículum, desarrollo personal y desarrollo social), subcategorías, códigos, respectivas frecuencias y porcentajes de los participantes en la investigación. En la Tabla 5 quedan recogidos los datos de los estudiantes-servicio y estudiantes-asignatura. 


\begin{tabular}{|c|c|c|c|c|}
\hline Categorías & Subcategorías & Códigos & $f$ & $\%$ \\
\hline \multirow{5}{*}{ Currículum } & Dominio contenidos & CURDO1 & 25 & 89,28 \\
\hline & Actitudes hacia el aprendizaje & CURAA2 & 21 & 75 \\
\hline & $\begin{array}{l}\text { Actitudes positivas hacia el } \\
\text { trabajo }\end{array}$ & CURPT3 & 20 & 71,42 \\
\hline & $\begin{array}{l}\text { Conocer las opciones de la } \\
\text { titulación }\end{array}$ & CUROT4 & 12 & 42,85 \\
\hline & $\begin{array}{l}\text { Ideas realistas sobre el mundo } \\
\text { del trabajo }\end{array}$ & CURIT5 & 10 & 35,71 \\
\hline \multirow{7}{*}{$\begin{array}{l}\text { Desarrollo } \\
\text { personal }\end{array}$} & Responsabilidad & DEPRE1 & 23 & 82,14 \\
\hline & Autoestima y autoeficacia & DEPAA2 & 19 & 67,85 \\
\hline & Trabajo en equipo & DEPTE3 & 18 & 64.28 \\
\hline & Superación & DEPSU4 & 16 & 57,14 \\
\hline & Motivación & DEPMO5 & 13 & 46,42 \\
\hline & Comunicación & DEPCO6 & 12 & 42,85 \\
\hline & Creatividad & DEPCR7 & 5 & 17,85 \\
\hline \multirow{5}{*}{ Desarrollo social } & Prosocialidad & DESPR1 & 23 & 82,14 \\
\hline & Intervención en la comunidad & DESIN2 & 22 & 78,57 \\
\hline & Diversidad y valores & DESDI3 & 17 & 60,71 \\
\hline & Convivencia & DESCO4 & 14 & 50 \\
\hline & Interacción & DESIN5 & 11 & 39,28 \\
\hline
\end{tabular}

Tabla 5. Resultados de los estudiantes.

Los estudiantes han considerado que esta metodología, desde el punto de vista curricular, les ha permitido un mayor dominio del contenido de la asignatura y actitudes más positivas hacia el aprendizaje y el trabajo. Para estas categorías plantean que "...refuerza bastante estudiar el contenido teórico de la asignatura y ver su aplicación en la realidad del aula" [CURDO1 \{19:4\}] y "...facilita el estudio de la asignatura al ser los propios compañeros los que cuentan las fases de la puesta en práctica en el CEIP de la metodología estudiada en la Facultad" [CURDO1 \{21:2\}]. Para las subcategorías conocer las opciones de la titulación e ideas más realistas sobre el mundo del trabajo, coinciden estudiantes-servicio y estudiantes-asignatura al argumentar que al estar en contacto con el CEIP y sus profesionales se hacen una idea de las intervenciones que realiza un pedagogo en los centros educativos y les abre nuevas posibilidades de cara a las prácticas externas de la titulación y como salida profesional. "Ha sido muy positiva la experiencia pues creía que los pedagogos no teníamos nada que hacer en un centro de educación infantil y primaria" [CURIT5 $\{4: 0\}]$.

Para la categoría desarrollo personal, esta metodología ha despertado su capacidad de asumir responsabilidades serias, ha aumentado la autoestima y autoeficacia, incrementa habilidades de trabajo en equipo, fomenta su deseo de superación ante todo tipo de dificultades, aumenta la motivación, mejora la comunicación y despierta la capacidad creativa de los alumnos. "El haber aceptado el voluntariado en el CEIP me ha servido para comprobar que puedo trabajar con profesores y compañeros y me ha motivado mucho pues creía que no me gustaba la carrera elegida” [DEPMO5 \{3:1\}]. 
"Al tener que trabajar con alumnos reales me he dado cuenta que puedo resolver problemas y dificultades por mi misma” [DEPAA2 $\{6: 2\}]$.

Para la categoría desarrollo social plantean que favorece la prosocialidad, sitúa al alumno en contacto con el entorno para su intervención, actitud más positiva hacia la apreciación de la diversidad y valores fundamentales, genera posibilidades para interactuar y convivir con otros compañeros y con adultos y permite la interacción con instituciones y agencias públicas y privadas. "Este voluntariado me ha gustado mucho pues he podido ayudar a personas que lo necesitan más que yo" [DESPR1 \{2:0\}]. "Para el curso que viene voy a apuntarme para ir al centro a ayudar pues todo lo que cuentan mis compañeros sobre lo que hacen en el CEIP me parece estupendo" [DESIN2 $\{13: 5\}]$.

En la Tabla 6 mostramos los datos de los profesores.

\begin{tabular}{|l|l|l|c|c|}
\hline \multicolumn{1}{|c|}{ Categorías } & \multicolumn{1}{|c|}{ Subcategorías } & Códigos & $\boldsymbol{f}$ & \% \\
\hline \multirow{4}{*}{ Currículum } & Articulación de los contenidos & CURAR1 & 22 & 100 \\
\cline { 2 - 5 } & Calidad de los aprendizajes & CURCA2 & 21 & 95,45 \\
\cline { 2 - 5 } & Estrategias didácticas & CURES3 & 20 & 90,90 \\
\cline { 2 - 5 } & Participación activa & CURPA4 & 18 & 81,81 \\
\hline \multirow{3}{*}{$\begin{array}{l}\text { Desarrollo } \\
\text { personal }\end{array}$} & Responsabilidad & DEPDA1 & 21 & 95,45 \\
\cline { 2 - 5 } & Trabajo en equipo & DEPTR2 & 19 & 86,36 \\
\cline { 2 - 5 } & Motivación & DEPMT3 & 19 & 86,36 \\
\cline { 2 - 5 } & Liderazgo & DEPLI4 & 15 & 68,18 \\
\hline \multirow{3}{*}{$\begin{array}{l}\text { Desarrollo } \\
\text { social }\end{array}$} & Prosocialidad & DESSO1 & 22 & 100 \\
\cline { 2 - 5 } & Intervención en la comunidad & DESIT2 & 22 & 100 \\
\cline { 2 - 5 } & Diversidad y valores & DESVA3 & 14 & 63,63 \\
\hline
\end{tabular}

Tabla 6. Resultados de los docentes.

En general, todos los profesores consideran esta metodología altamente positiva y han notado mejores calificaciones y un cambio en la actitud en los estudiantes. "Cada quince días los estudiantes-servicio han presentado oralmente sus informes sobre su intervención en el CEIP y esos días el resto de los compañeros de clase han preguntado más dudas y se han implicado más en el desarrollo de la asignatura" [CURPA4 $\{2: 0\}]$. "Los estudiantes se han preparado la teoría antes de ir a al centro pues debían conocerla para su puesta en práctica" [CURAR1 \{11:3\}].

Además ha aumentado la asistencia, participación y liderazgo de los estudiantesservicio en las clases teóricas de la Facultad, "...las clases estaban atestadas de estudiantes cuando en años anteriores la asistencia era mucho menor" [CURPA4 $\{2: 0\}]$. Esto implica mayor responsabilidad y aumento de la motivación.

Uno de los inconvenientes planteado, aunque no aparece recogido como queja, es que esta metodología requiere un esfuerzo adicional a su trabajo diario en las clases. "No sólo debemos preparar nuestras clases sino orientar a los estudiantes-servicio y 
profesionales del CEIP para que exista coordinación y además revisar todos los informes que los estudiantes-servicio deben presentar en la Facultad" [CURAR1 $\{2: 0\}]$.

Los profesores, igualmente, han valorado la importancia que el ApS aporta al desarrollo social de los estudiantes favoreciendo la prosocialidad, mayor intervención en la comunidad y la consideración de las diferencias entre personas.

Para el representante de la Universidad en la categoría currículum ha destacado una formación integral, mayor preparación profesional, participación activa y puesta en práctica de nuevas estrategias didácticas. En la categoría desarrollo personal, responsabilidad y prosocialidad y en la categoría desarrollo social, mayor intervención en la comunidad, fortalecimiento de las relaciones con otras instituciones y sentido de pertinencia, por este orden.

\section{Conclusiones}

Muchos países están promoviendo prácticas de ApS en el sistema educativo desde hace bastante tiempo. En algunos, estas prácticas se están introduciendo como obligatorias en la Educación Secundaria y Universidad (Holanda, Argentina o USA), al comprobar los resultados positivos tanto en términos de éxito académico como de compromiso social.

En esta misma línea, consideramos que el diseño de nuestro plan de acción ha permitido la incorporación, con muy buenas perspectivas, de la metodología ApS en la asignatura de Didáctica General y, por tanto, ha quedado cubierto el primer objetivo.

Podemos concluir igualmente, tras el análisis de los resultados, que se ha cumplido el segundo objetivo. Todos los sectores implicados en la investigación están convencidos que el ApS es una buena estrategia metodológica que favorece el desarrollo curricular, personal y social.

\section{Estudiantes-servicio y estudiantes-asignatura}

Los estudiantes han corroborado en las entrevistas que esta metodología permite vincular los contenidos teóricos a la práctica, promueve una mayor formación práctica y la reelaboración de los contenidos teóricos para hacerlos más pertinentes al contexto social para el desempeño profesional. Los nexos académicos con el currículum de la asignatura han sido muy claros y se ha combinado el servicio a la comunidad con el aprendizaje de contenidos, habilidades y valores. En definitiva, consideran que ha habido integración del aprendizaje académico y éste ha sido la clave del éxito del ApS.

En el ámbito personal, al diseñar proyectos, realizarlos, tomar decisiones, resolver problemas, presentar los proyectos, hablar en público, sentirse útiles y reconocidos eleva a niveles importantes su responsabilidad, afán de superación, motivación, comunicación, creatividad y autoestima y confianza. 
Por último, al realizar acciones sociales sin la búsqueda de recompensas materiales los estudiantes han valorado mucho más esta experiencia pues, en definitiva, aumenta la probabilidad de generar una reciprocidad positiva de calidad en las relaciones interpersonales y sociales, mejorando su identidad.

\section{Profesorado Facultad y Profesores Centro de Educación Infantil y Primaria}

Los profesores han confirmado un aumento de la satisfacción respecto del mejoramiento de la articulación y calidad en los aprendizajes, puesta en práctica de nuevas estrategias metodológicas y una participación activa de los estudiantes en el proceso educativo, al ser una metodología pedagógica que entra dentro de la filosofía de la educación experiencial. Además de estar activamente implicados en el propio proyecto, los estudiantes han tenido la oportunidad de seleccionar, diseñar, poner en práctica y evaluar su actividad de servicio.

Consideran que este tipo de aprendizaje por observación que realizan los estudiantes a través de la metodología de ApS tiene un gran impacto en su modelo de comportamiento y está más cercano a su futuro ejercicio profesional. Con esta metodología se enfrentan a modelos y comportamientos regulados por determinados valores y contravalores que inciden igualmente en su aprendizaje personal, social y ético.

Estos resultados están en la misma línea que las investigaciones desarrolladas en Estados Unidos, Latinoamérica y Europa al mostrar el impacto positivo de las prácticas de ApS en la calidad de los aprendizajes académicos, así como en la formación humana y social de los futuros profesionales.

Además, hemos constatado por las actas de los últimos cursos de esta asignatura que el número de no presentados y suspensos ha descendido considerablemente. En concreto, el descenso ha sido de un $22 \%$ para los no presentados y un $17 \%$ de los suspensos. Estos datos deben ser tomados con cautela hasta no contrastar los resultados de ApS con los obtenidos con otra metodología para poder establecer si existen diferencias significativas.

\section{Representante de la Universidad}

Para la Universidad el ApS permite la formación integral de los estudiantes e impulsa el compromiso con la comunidad. Por ello, debe instar a los docentes a incorporar estrategias didácticas que integren adecuadamente la preparación para la práctica profesional y para el ejercicio de responsabilidad social de sus estudiantes y titulados. Además de considerar las titulaciones como ejes de cambio como plantea Rué (2009). Esta estrategia metodológica refuerza los lazos sociales mediante el trabajo común y en equipo y con la mejora de la convivencia entre todos los ciudadanos. 


\section{Propuestas de mejora}

En la autoevaluación realizada para detectar las posibilidades de institucionalización y mejoras pedagógicas del ApS hemos comprobado que debemos implantar y fortalecer docencia estructurada de primer, segundo y tercer ciclo (itinerarios de grados, programas de doctorado, títulos propios,...) y crear asignaturas de libre elección, dotando a las existentes de contenidos teóricos y prácticos aplicables al ApS. Entre las mejoras relacionales, el conocimiento, implicación y compromiso en proyectos por parte de los individuos y grupos participantes; y la colaboración interinstitucional. La Universidad debe ser la encargada de establecer convenios con entidades sociales e impulsar instancias que faciliten la difusión de ideas, la presentación de las propuestas que han tenido éxito y la ayuda a la coordinación entre instituciones. Igualmente permitir la interactividad social mediante la utilización de recursos tecnológicos, tanto para agilizar el acceso a la información, la comunicación entre estudiantes y profesores, entre Universidades, entre países y con otros agentes sociales, como para el desarrollo de la formación en plataformas virtuales de enseñanza para multiplicar el efecto de las acciones formativas desarrolladas. Y entre las mejoras organizativas, una mayor planificación y una programación más definida de cada acción formativa y una duración y flexibilidad más adecuada.

Para finalizar comentar que, si bien el ApS es una metodología educativa, una herramienta pedagógica que vincula la teoría con la práctica, en esta investigación ha sido valorada también como herramienta de desarrollo comunitario, de cohesión de la comunidad, de compromiso responsable (López Herrerías, 2010). El ApS ha funcionado no solamente para sostener la motivación y el proceso de formación de los estudiantes universitarios implicados sino también como motor de superación de las dificultades, de activación de redes sociales y de fomento de la confianza de los ciudadanos (Batlle, 2011; García y Mínguez, 2011).

\section{Referencias bibliográficas}

ANNETTE, J. (2000). Civic Participation and Education for Citizenship. Comunicación presentada en Political Studies Association, UK 50th Annual Conference, Londres.

ANNETTE, J. (2005). Character, civic renewal and service learning for democratic citizenship in higher education. British Journal of Educational Studies, 53(3), 326340.

BARRIO, J.L. (2005). La transformación educativa y social en las comunidades de aprendizaje. Teoría de la Educación. Revista Interuniversitaria, 17, 129-156.

BATLLE, R. (2011) ¿De qué hablamos cuando hablamos de aprendizaje servicio?. Crítica, 972, 49-54.

BATlle, R. (2012). Impulso del Aprendizaje-Servicio en España. Panorama 2012. Recuperado de http://www.slideshare.net/rbatlle/panorama-del-aps-en-espaa-2012 (Consultado el 12 de diciembre de 2012). 
BEDNARZ, S., CHALKLEY, B., FLETCHER, S., HAY, I., LE HERON, E., MOHAN, A., \& TRAFFORD, J. (2008). Community Engagement for Student Learning in Geography. Journal of Geography in Higher Education, 32(1), 87-100.

BUCHANAN, A.M., BALDWIN, S., \& RUDISILL, M. (2002). Service Learning as Scholarship in Teacher Education. Educational Research, 31(8), 30-36.

BUTIN, D. W. (2006). The limits of service-learning in higher education. The Review of Higher Education, 29, 473-498.

CAMPUS COMPACT EDUCATING CITIZENS-BUILDING COMMUNITIES. Recuperado de http://www.campuscompact.org/ (Consultado el 30 de octubre de 2012).

CENTRO LATINOAMERICANO DE APRENDIZAJE SERVICIO SOLIDARIO (CLAYSS) (2012). Recuperado de http://www.clayss.org.ar/ (Consultado el 28 de diciembre de 2012).

FURCO, A. (2005). Impacto de los proyectos de aprendizaje-servicio, en Programa Nacional Educación Solidaria. Unidad de Programas Especiales Ministerio de Educación, Ciencia y Tecnología. Aprendizaje y servicio solidario en la Educación Superior y en los sistemas educativos latinoamericanos. Actas del 7mo. Seminario Internacional Aprendizaje y Servicio Solidario. República Argentina, 19-26.

FURCO, A., \& BILling, S. H. (2002). Service Learning. The Essence of the Pedagogy. Greenwich: Information Age Publishing.

GALAMBOS, R., \& KOZMA, J. (2005). Service-Learning and Civic Education in Hungarian Higher Education. Vocational Education: Research and Reality, 10, 4051 .

GARCÍA, A., \& MÍNGUEZ, R. (2011). Los límites de la educación en valores cívicos: cuestiones y propuestas pedagógicas. Educación XX1, 14(2), 263-285.

GÜRTLER, L., \& HUBER, G. L. (2007). Should we generalize? Anyway, we do it all the time in everyday life. En Gürtler, L., Mechthild, K,. \& Huber, G.L. (eds.), Generalization in Qualitative Psychology, 17-35. Tübingen: Verlag Ingeborg Huber.

HOLDSWORTH, C., \& QUINN, J. (2010). Student volunteering in English higher education. Studies in Higher Education, 35(1), 113-127.

HOLLANDER, E. L., SALTMARCH, J., \& ZLOTKOWSKI, E. (2001). Indicators of Engagement. En Simon, L. A. et. al. (eds.), Learning to Serve: Promoting Civil Society Through Service-Learning, 45-61. Norwell: Kluwer Academic Publishers.

INTERNATIONAL ASSOCIATION FOR RESEARCH ON SERVICE-LEARNING AND COMMUNITY ENGAGEMENT (2012). Recuperado de http://www.researchslce.org/ (Consultado el 3 de enero de 2013). 
LEARN AND SERVE AMERICA (2012). Recuperado de http://www.learnandserve.gov/ (Consultado el 29 de diciembre de 2012).

LÓPEZ HERRERÍAS, J.A. (2010). Educación de calidad y en competencias para la competitividad o para la cooperación. Revista Complutense de Educación, 21(1), 107-122.

MANZANO, V. (2010). El modelo de aprendizaje-servicio y su potencial para la educación superior. Conferencia inaugural de las VI Jornadas de docencia en Psicología. Universidad de Sevilla, 15 de junio.

MARTÍNEZ, M. (2007). Formación para la ciudadanía y educación superior. Revista Iberoamericana de Educación, 42. Recuperado de www.rieoei.org/boletin42_5.htm (Consultado el 11 de noviembre de 2012).

MARTÍNEZ-ODRÍA, A. (2005). Voluntariado y Service-Learning en la escuela. Tesis doctoral, Universidad de Navarra.

MURPHY, T. (2010). Conversations on engaged pedagogies, independent thinking skills and active citizenship. Issues in Educational Research Special edition on service learning, 20(1), 39-46.

RED ESPAÑOLA DE APRENDIZAJE-SERVICIO (2012). Recuperado de http://www.aprendizajeservicio.net/ (Consultado el 30 de noviembre de 2012).

REINDERS, H. (2010). The Promotion of Learning Processes through Service Learning in Higher Education. Zeitschrift Fur Padagogik. 56(4), 531-547.

RUÉ, J. (2009). El cambio en la Universidad, sus epistemologías y consecuencias de las mismas. Revista Complutense de Educación, 20(2), 295-317.

SERVICE-LEARNING RESEARCH AND DEVELOPMENT CENTER DE LA UNIVERSIDAD DE CALIFORNIA (2012). Recuperado de http://gse.berkeley.edu/research/slc/ (Consultado el 5 de diciembre de 2012).

TAPIA, Ma N. (2000). La Solidaridad como Pedagogía. Buenos Aires: Ciudad Nueva.

TAPIA, M ${ }^{\mathrm{a}}$ N. (2008). Aprendizaje y servicio solidario. Buenos Aires: Ciudad Nueva.

\section{Correspondencia con la autora:}

Margarita R. RODRÍGUEZ GALLEGO

Departamento de Didáctica y Organización Educativa

Facultad de Ciencias de la Educación

C/ Pirotecnia s/n. 41013 Sevilla

e mail: margaguez@us.es

Teléfono: (34) 955420618 